\title{
Influence of the shape of the electrodes on the tunnel current
}

\author{
R. J. P. Keijsers and J. Voets \\ Research Institute for Materials, University of Nijmegen, Toernooiveld 1, 6525 ED Nijmegen, The Netherlands \\ O. I. Shklyarevskii \\ Research Institute for Materials, University of Nijmegen, Toernooiveld 1, 6595 ED Nijmegen, The Netherlands \\ and \\ B. I. Verkin Institute for Low Temperature Physics and Engineering, National Academy of Sciences of Ukraine, \\ 47 Lenin ave., 310164 Kharkov, Ukraine \\ E-mail: olegs@sci.kun.nl \\ shklyarevskii@ilt.kharkov.ua \\ H. van Kempen \\ Research Institute for Materials, University of Nijmegen, Toernooiveld 1,6525 ED Nijmegen, The Netherlands \\ E-mail: hvk@sci.kun.nl \\ Received April 27, 1998
}

\begin{abstract}
The tunnel resistance of highly stable, mechanically controlled break junctions of $\mathrm{Al}, \mathrm{Au}, \mathrm{Cu}, \mathrm{Pb}$, $\mathrm{Ni}$, Pt, and Pt-Ir, have been recorded as a function of the electrode spacing over 6-7 decades. Clear deviations from the expected exponential behavior have been observed. Comparison with previous experimental and theoretical studies indicate that the discussed deviations in some cases are most probably due to the shape of, rather than to interactions between, the two electrodes.
\end{abstract}

PACS: $73.40 . \mathrm{Gk}$

\section{Introduction}

Over the last decade, the scanning tunneling microscope (STM) has developed into one of the most generally used instruments for the study of surfaces and small structures [1-5]. The atomic resolution in topographical measurements is a consequence of the strong dependence of the tunnel current on the distance between the tip and sample. The simplest and crudest approximation describing the tunnel current is a planar model of the barrier, leading to an exponential decrease of the tunnel current as a function of increasing electrode spacing. If the interaction between the tunneling electron and the opposing electrode, at which a charge builds up, is incorporated as a first order correction (image potential), the barrier height and width are found to reduce, but the general result stays the same for low bias voltages [6].

Some experimental investigations of the tunnel current as a function of distance between tip and sample have been presented in the past. Gimzewski and Möller [7] have studied the transition from tunneling regime to point contact for a clean Ir tip and polycrystalline Ag surface. They observed the onset of a plateau in the tunnel resistance at small tip-sample distances $(\leq 2.5-3 \AA$ before a jump to contact), which was subsequently explained theoretically by Lang [8]. He modeled the system by two flat jellium electrodes with a single tip atom present at one of the surfaces, and found that the tunnel current as a function of electrode separation leveled out at a conductance value of $\eta 2 e^{2} / h(\eta$ being of order unity) as the situation came close to a one-atom size contact. A tight-binding calculation by Ferrer, Martín-Rodero, and Flores [9] produced comparable results. Düring, Züger, and Pohl [10] studied the approach of an Ir tip to an Ir sample, and found only a weak onset for a plateau at small separations. Ciraci and Tekman [11] showed theoretically that, depending on material and tip-specific features, the tunnel resistance may saturate at very small distances, but can also increase almost 
linearly up to contact when plotted on a logarithmic scale. On the other hand, Krans et al. [12] observed faster than exponential behavior at a Pt mechanically controllable break junction, which they explained from attractive forces between the front atoms of the two tips. However, in all these experimental and theoretical investigations of tipsample approach, deviations from exponential tunneling behavior were observed over at most two decades of the tunnel resistance, just before the jump to contact.

In a recent theoretical article, Laloyaux et al. [13] calculated the tunnel current for different $3 D$ geometries of the two electrodes (tip and sample) by solving the exact Schrödinger equation numerically for the electron tunneling problem in such a multidimensional configuration. They studied the tunnel current as a function of tip-sample distance for sharp (hemispherical) and blunt (cylindrical) tips above a flat surface, and also studied the influence of the presence of a Gaussian boss or dip at the sample surface. Their results showed that certain tip-sample geometries can lead to significant deviations from the exponential relation between tunnel current and tip-sample distance over more than four orders of magnitude of the tunnel current, whereas the above described electron-mediated tipsample interactions, which arise because the potential barrier between the electrodes allows the wave functions to overlap at very small separations [14], have never been predicted nor observed to cause deviations for more than two orders of magnitude.

In this work we shall present experimental observations of deviations from exponential tunneling behavior over a wide range of tunnel resistances between $10-20 \mathrm{k} \Omega$ and 1-5 G $\Omega$, which can be attributed to a dependence of the tunnel current vs. distance relation on the $3 D$ junction geometry. The results will be compared to the theoretical results of Laloyaux et al. [13]. To ensure that the tip in our experiments had a well-defined apex, we discarded all measurements which did not show a well-defined jump to a one-atom point contact at relatively small distances. We also observed current-distance curves which showed characteristics attributable to strong mutual interactions between the electrodes. These last results are discussed in detail elsewhere [15].

\section{Experimental}

The observation of possible deviations from exponential tunneling behavior requires a highly stable tunnel junction with an adjustable tip-sample (or tip-tip) distance. Therefore, we used the mechanically controllable break (MCB) junction tech-

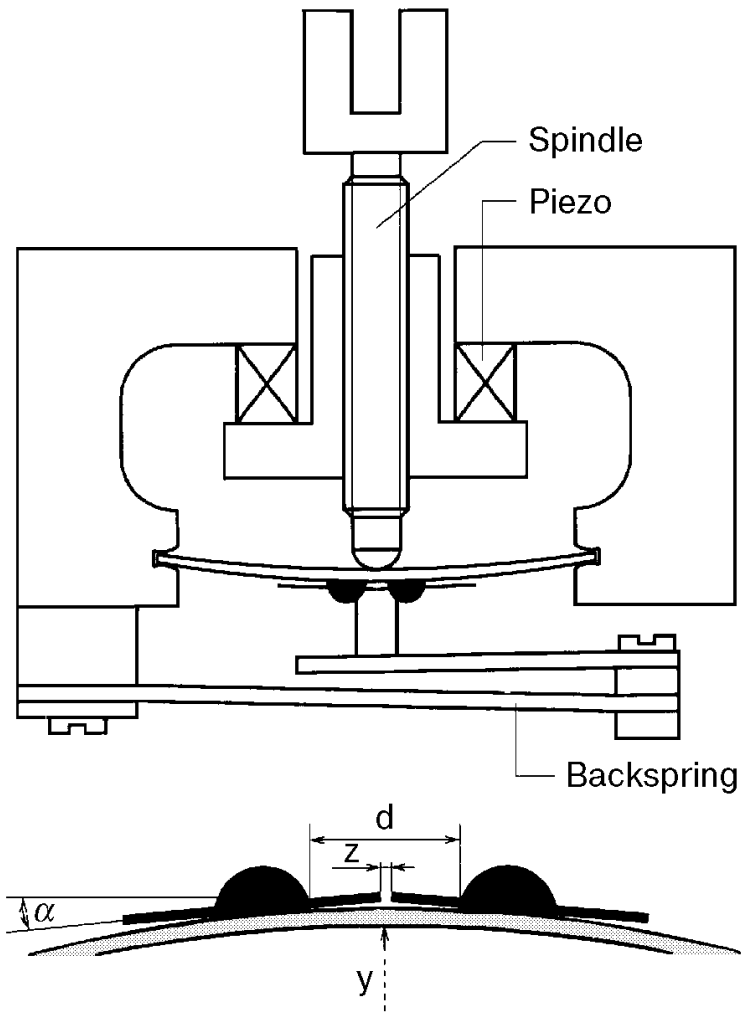

Fig. 1. Schematic drawing of the MCB-setup. The sample consists of a thin $(50 \mu \mathrm{m})$ metallic wire, which is glued with two drops of Stycast 2850 FT on top of a phosphorbronze bending beam that is covered with an insulating layer. The wire is cut to more than $80 \%$ of its diameter, and can be broken in vacuum at low temperatures by turning the spindle. A differential screw mechanism that is also attached to this spindle can be used to bring the junction into the tunnel regime, or to establish a small contact. Very small tip-tip displacements can then be realized in a controlled way by applying a voltage over the piezo ring. The backspring can be used to obtain «negative» bending angles, but was also found to increase the stability of the setup strongly.

nique [16] for our experiments. $\mathrm{MCB}$ junctions have already proven their high stability and adjustability in a study of the transition from weak link to superconducting tunnel junction [16] and in oneatom point contact experiments [12]. The stability of our setup is illustrated by the fact that we found it possible to reproduce current-distance characteristics several times over periods of more than $60 \mathrm{~min}$. A drawback of the MCB-technique is that it is difficult to calibrate the tip-sample displacement, because it depends on the specific sample arrangement and on the way the sample breaks.

The setup we have used is drawn in a somewhat simplified way in Fig. 1. The sample (a $50 \mu \mathrm{m}$ metallic filament) is glued onto a phosphor-bronze bending beam covered with a thin insulating layer 
of kapton foil, using two small drops of Stycast 2850 FT epoxy. The sample is then cut for about $90 \%$ of its diameter at the midpoint between the two drops of epoxy. After that, the sample is mounted in the setup (Fig. 1), and is cooled to $4.2 \mathrm{~K}$ in high vacuum surroundings. The sample is then broken by turning the spindle, thus creating two clean electrodes. The tunnel distance (or contact size) can be adjusted using a differential screw connected to the spindle. Both spindle and differential screw are driven by using fork-blade mechanisms, enabling us to decouple the setup from the outside world if desired. Fine adjustment and scanning of the distance is done by means of the piezo driver. Mounting of a backspring (see Fig. 1) turned out to increase the stability of our setup strongly. External vibrations were suppressed by suspending the cryostat from the ceiling using elastic cords («bungy cords»)

In order to be able to observe deviations from exponential behavior of the tunnel resistance $R_{T}$ as a function of the tip distance $z$, we must ascertain that $z$ is proportional to the vertical displacement $y$ of the center of the beam when it is bent. Therefore, the relation between $z$ and $y$ will be discussed in considerable detail here.

When the beam is bent, the surface of the beam beneath the filament will be stretched, leading to an increase in the distance between the two fixation points, and thus to an increase of $z$. Assuming that the tips just touch when there is no vertical displacement, the increase $\Delta_{s}$ due to surface stretching can be expressed as [17]

$$
\Delta_{s}=\frac{F h}{16 E I}\left(2 l d-d^{2}\right), \quad I=\frac{w h^{3}}{12} .
$$

Here, $d$ is the distance between the two anchoring points (see Fig. 1), $F$ is the vertical force exerted on the bending beam, $E$ is the modulus of elasticity of the beam, and $h, l$, and $w$ are its thickness, length and width. Using an expression for the vertical displacement from Ref. 17, $\Delta_{s}$ can be rewritten as

$$
y=\frac{F l^{3}}{48 E I} \Rightarrow \Delta_{s}=3 y \frac{h d}{l^{2}}\left(2-\frac{d}{l}\right) .
$$

The beams we use have dimensions $l \times w \times h=$ $=28 \times 10 \times 0.4 \mathrm{~mm}$, and the distance $d$ is about $0.5 \mathrm{~mm}$.

Bending of the beam also leads to a non-zero slope $\alpha$ at the fixation points (Fig. 1). This causes an additional contribution $\Delta_{z}$, to the distance between the tips, as well as a vertical displacement $\Delta_{y}$

$$
\Delta_{z}=d(1-\cos \alpha)+2 p \sin \alpha, \Delta_{y}=2 \varepsilon \sin \alpha,
$$

where $\varepsilon$ stands for the distance between the point where the wire has been broken and the middle of the section of the wire between the two anchoring points, and $p$ is the vertical distance of the wire at the anchoring points to the plane of flexure. For the case of a wire lying on top of a bending beam, this distance will be half the beam thickness $h$. The slope $\alpha$ is given by [17]

$$
\alpha=\frac{3 y d}{l^{2}}\left(2-\frac{d}{l}\right)
$$

and has a small value in all experiments $(\alpha=$ $=0.008 \mathrm{rad}$ for $y=2 \mathrm{~mm}$ ). Therefore, $\Delta_{z} \approx$ $\approx(1 / 2) d \alpha^{2}+2 p \alpha$. For $p=h / 2$, the second term equals $\Delta_{s}$. The term $(1 / 2) d \alpha^{2}$ is proportional to $y^{2}$, which in principle leads to a nonlinear relation between the separation $z=\Delta_{s}+\Delta_{z}$, of the two tips, and $y$. However, in our setup, this term is two orders of magnitude smaller than both the other term in (3) and $\Delta_{s}$. In an experiment, $y$ is typically varied over less than $1 \mu \mathrm{m}$ around a value of $2 \mathrm{~mm}$, and the change in slope $\partial z / \partial y$ over this range is less than $1.5 \cdot 10^{-4} \%$. Neglecting the nonlinear term, a value of 330 for the ratio $y / z$ is found, which is the estimated attenuation of the applied vertical displacement to the induced tip-tip distance.

The vertical displacement $\Delta_{y}$ is zero when the wire is cut exactly in the middle $(\varepsilon=0)$, but can be quite large when the wire breaks close to one of the fixation points $(\varepsilon=d / 2)$,

$$
\Delta_{y} \approx 2 \varepsilon \alpha=3 y \frac{d^{2}}{l^{2}}\left(2-\frac{d}{l}\right),
$$

which is of the same order of magnitude as $z$. Because $\Delta_{y} \propto y$, the distance between the two tips will nevertheless be proportional to $y$, albeit the tips do not approach horizontally. However, when preparing the samples, extreme care was taken to ensure that the notch was as close as possible to the midpoint between the two gluing points, leading to no or just a small vertical displacement of the electrodes with respect to each other.

\section{Results and discussions}

In the above it has been shown that bending of the beam leads to $z \propto y$. There are however other effects that may affect the shape of measured current-distance characteristics. First of all, the experimentally measured $R_{T}(z)$ curve may deviate from its real shape due to thermal drift. However, we found that this effect was only significant in our experiments during the first hour after filling our cryostat with liquid helium. 

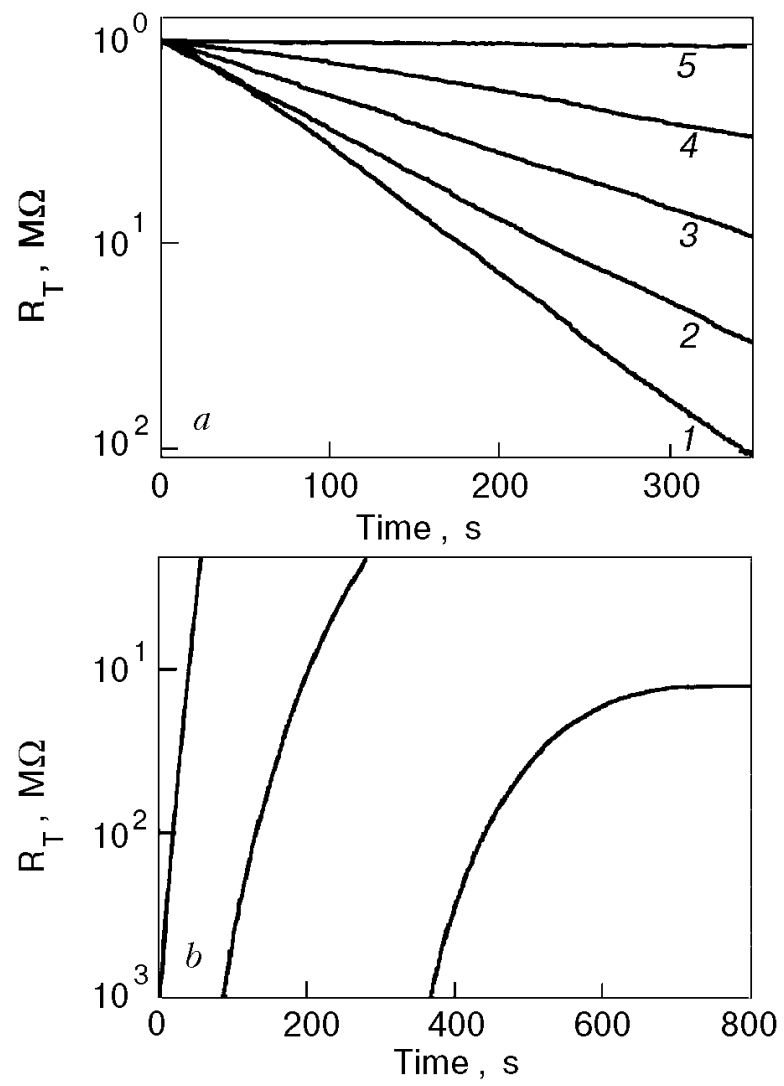

Fig. 2. (a) Drift of the tunnel resistance $R_{T}$ of an Al-sample directly after break $(T=4.2 \mathrm{~K})$. Each curve represents a period of about $6 \mathrm{~min}$, starting $10(1), 20(2), 30(3), 60(4)$ and 180 (5) min after breaking. After three hours, the change in $R_{T}$ has become less than $5 \%$ over a period of 6 min. (b) Drift of the tunnel resistance $R_{T}$ of a $\mathrm{Pb}$-sample, measured directly after the electrodes have been pressed together for a short time ( $T=$ $=4.2 \mathrm{~K}$ ). The drift decreases quickly and is negligible after $12 \mathrm{~min}$.

Furthermore, we found that material flow («backward», i.e., the tunnel distance increases while the piezo voltage is kept constant) was a rather large effect shortly after breaking the sample. It was found to depend strongly on the notch depth, and turned out to be larger for «soft» materials like $\mathrm{Au}, \mathrm{Al}$ and $\mathrm{Pb}$, than for $\mathrm{Ni}, \mathrm{Pt}$ or a Pt-Ir(3\%) alloy. Figure 2, $a$ shows the drift of the tunnel resistance $R_{T}$ for an Al-sample which was previously cut for about $80 \%$ of its diameter. The change in $R_{T}$ was as large as two orders of magnitude over 5-6 min (which is the typical measuring time) shortly after the break, but dropped to an acceptable level of a linear change of $R_{T}(t)$ of $2-5 \%$ in $6 \mathrm{~min}$ after waiting for $3 \mathrm{~h}$, and became negligible after a period of $10 \mathrm{~h}$. The total «backflow» of sample material can be estimated to be $\approx 100-$ $1000 \AA$, starting from the moment of break. The amount of wire elongation shortly before the break is approximately $5-10 \mu \mathrm{m}$.

Another material-flow effect can be observed when one is changing the tip shapes by making a deep excursion of the electrodes into each other, creating a point contact with a diameter of $100-$ $300 \AA$ for a short time. The deformation is at least partially elastic, and after separating the electrodes $R_{T}$ tends to decrease (see Fig. 2,b). However, this effect is much smaller than the one described above, and $R_{T}$ saturates after $10-15$ min for «soft» materials, and after 3-5 min for Pt and the $\mathrm{Pt}-\operatorname{Ir}(3 \%)$ alloy.

A flow of the bending beam material may also be involved in the effects observed, but its contribution must be very small due to the fact that the cross-sectional area of the bending beam is about four orders of magnitude larger than the one of the notched filament, and almost all of the bending takes place within the elastic deformation limits of the beam.

After a jump to a one-atom contact, the electrodes can also suffer an irreversible change in atomic configuration. In that case, however, a new equilibrium situation is reached within seconds.

All the effects described above can influence the shape of the measured current-distance characteristics. However, by waiting long enough for electrode material to relax into an equilibrium configuration, it was possible to diminish these effects to a negligibly small magnitude.

It was shown above that, theoretically, the transfer from vertical displacement to tip-tip displacement should not deviate noticeably from linear behavior. In Fig. 3, $a$, the tunnel resistance $R_{T}$ of $\mathrm{Pt}, \mathrm{Au}$ and $\mathrm{Cu}$ MCB-junctions is plotted on a logarithmic scale as a function of the applied piezo voltage, which in turn is proportional to the vertical displacement $y$. All curves are displayed «as recorded», demonstrating the high stability of the MCB setup; the curves were always reproduced 2-4 times to check their validity. All three curves of Fig. 3, $a$ display a perfect exponential behavior of the tunnel resistance over about seven orders of magnitude, at bias voltages of $1 \mathrm{mV}$ below, and $100 \mathrm{mV}$ above, $R_{T}=10^{10} \Omega$. The fact in itself that it is possible to record perfect exponential behavior strongly supports the assumption of a linear $z(y)$ dependence, because it is very unlikely that some nonlinear relation between $\mathrm{y}$ and the tip-tip distance can be compensated so perfectly by effects of the shape of, or interactions between, the two electrodes. An exponential $R_{T}(z)$ dependence can be the result of a tunnel geometry of a sharp tip above 

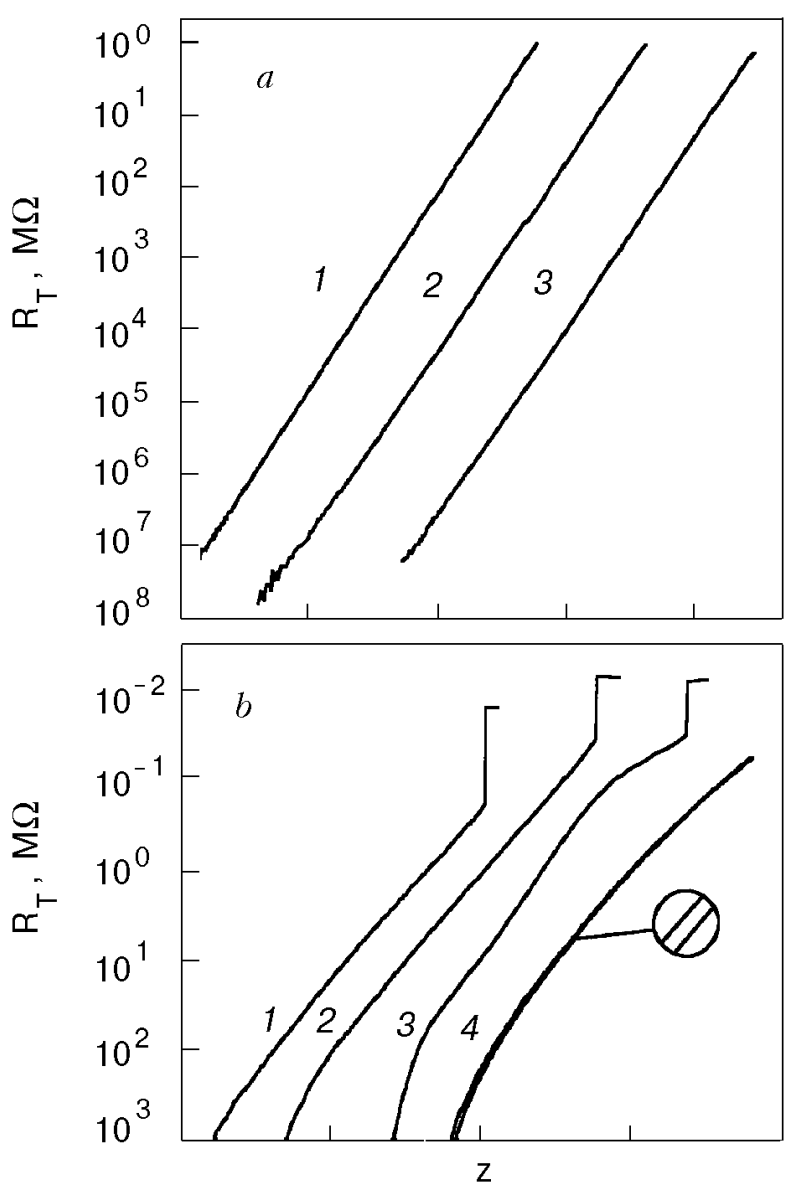

Fig. 3. (a) Logarithmic plot of the tunnel resistance as a function of $z$-piezo voltage for MCB-junctions of $\mathrm{Pt}(1), \mathrm{Au}$ (2), and $\mathrm{Cu}$ (3), at $T=4.2 \mathrm{~K}$. Curves $1-3$ show almost perfect exponential behavior over at least seven orders of magnitude; $(b)$ Logarithmic plot of the tunnel resistance as a function of $z$-piezo voltage for MCB-junctions of $\mathrm{Al}(1), \mathrm{Pt}(2), \mathrm{Pt}-\mathrm{Ir}(3 \%)$ (3) and $\mathrm{Pb}(4)$, at $T=4.2 \mathrm{~K}$. Curves $1,2,4$ display a slow bending (decrease of the slope) over the same range. Curve 3 shows on some parts an increase, and on some parts a decrease of the slope. All these types of behavior were predicted theoretically by Laloyaux et al. [13]. The curves were recorded starting from a large distance (high $R_{T}$ ), decreasing the electrode spacing until a jump to contact took place. For curve 4 , the scans were reversed shortly before the jump, showing the small piezo-hysteresis in our setup. A small part of curve 4 has been enlarged by a factor ten. The achieved displacement is estimated to be $\approx 1 \AA$ per decade change in $R_{T}$. Because the electrode separation to piezo voltage ratio depends on specific sample parameters (see text), the horizontal axis had to be rescaled for some curves for clarity.

a flat surface, for it was shown that a geometry of a hemispherical tip above a flat surface does not lead to notable deviations from exponential behavior [13], and furthermore, attractive Van der Waals forces are negligible for sharp conical tips [14]. The applied bias voltages are sufficiently low to avoid effects of power dissipation [7] and electrostatic forces [10]. We also recorded $R_{T}(z)$ curves at bias voltages up to $300 \mathrm{mV}$, and found no significant changes compared to the measurements at lower biases.

When all precautions are taken, the $R_{T}(z)$-curves measured when scanning $z$ from a large distance almost up to the jump-to-contact point, and then scanning back, practically coincide. The very small deviation between the «upwards» and «downwards» recorded curves, which is due to the hysteresis of the piezo driver, is hardly visible. (The loops could be reproduced several times, so the deviations cannot be due to material flow.)

As stated before, it is not possible to calibrate the electrode separation as a function of applied piezo voltage for an MCB-junction, because the $z(y)$ depends on certain sample parameters (e.g. the distance $d$ between the gluing points, see formula (2)). Indeed we found that the slope of $\log \left(R_{T}(z)\right)$ as a function of piezo voltage may differ by a factor of two for different samples. However, using known values for the work functions $\varphi$ of specific materials for clean electrodes, and a simple formula for the tunnel current as a function of tip-sample separation [1], it can be estimated that a one-decade change in the value of the tunnel current (tunnel resistance) corresponds roughly to a displacement of about $1 \AA$ for the materials discussed here (and also for a number of other materials). This enables us to get a reasonable estimation of the experimentally obtained electrode displacements. The value is comparable to the ones that can be found from experiments with an Ir tip approaching a Ag surface [7] (1.7 $\AA$ per decade), an Ir tip moving towards an Ir surface [10] $(1.0 \AA$ 放 decade $)$, and experiments using a Pt MCB junction [12] (0.6-1.1 $\AA$ /decade).

The curves in Fig. 3, $b$ display features which, to our opinion, must be attributed to effects of tipsample geometries. They show clear deviations from an exponential dependence over the whole range that was scanned (more than four orders of magnitude in $R_{T}$, starting at $1 \mathrm{G} \Omega$ and ending at $\approx 100-$ $50 \mathrm{k} \Omega$, where a jump to a one atom point contact occurs). This suggests that it is unlikely that we are dealing with effects stemming from tip-sample interactions, because these have been shown to extend over a much smaller range close to contact, both theoretically (over one [8,9] or two [11] decades) and experimentally (up to only $20-30 \mathrm{k} \Omega[7,10]$ or $500 \mathrm{k} \Omega$ [12]). The observed deviations from an exponential dependence were present in about five percent of all recorded curves. The curves displayed 


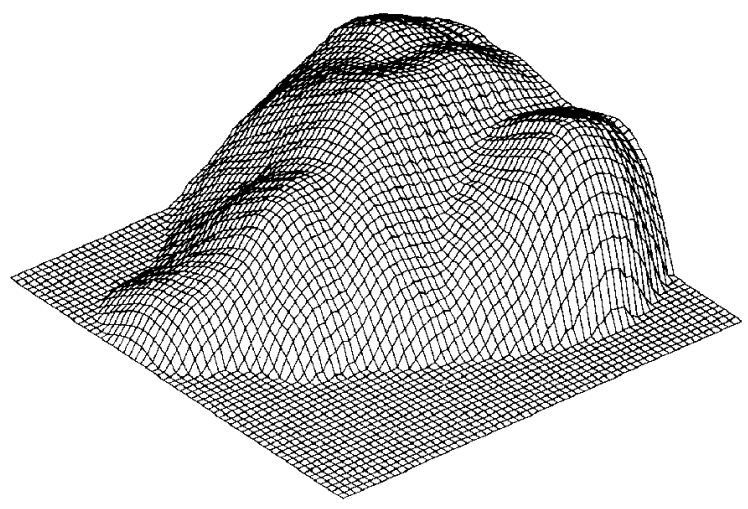

Fig. 4. Three-dimensional image of the foremost part of the surface of the bluntest Au electrode. All data below a certain value have been cut, and a horizontal plane has been introduced in the figure at this value. The figure displays an area of about $15 \times 15 \AA$.

in Fig. 3, $b$ have been selected to demonstrate clearly the observed deviations.

Curves 1,2 and 4 in Fig. 3,b display a gradual, continuous change of the slope over the whole range of displacement, starting at least $4-5 \AA$ before the jump to contact. This kind of behavior was predicted by Laloyaux et al. [13] for a geometry of a cylindrical tip facing a flat sample, where they found a slow bending of $\log \left(I_{T}(z)\right)$ over four orders of magnitude of the tunnel current for a tip-sample displacement from 1 to $5 \AA$. Curve 3 of Fig. $3, b$ displays another type of $R_{T}(z)$-dependence that we have observed. It is characterized by an alternately increasing and decreasing slope of $\log \left(R_{T}(z)\right)$, and was observed for $\mathrm{Al}$ and $\mathrm{Pt}$ also. This type of curve was predicted by Laloyaux et al. [13] for configurations of a hemispherical tip (modeling a sharp tip) opposing a gaussian dip or boss in or on a flat surface. Their calculated $\log \left(I_{T}(z)\right)$ showed approximately the same, though somewhat weaker, effects in the tip against dip geometry compared to the one of a tip facing a boss. Also, the tunnel current decreased more slowly for the first geometry when compared to the latter (three, respectively four orders of magnitude at a displacement from 1 to $5 \AA$ ). We indeed did observe that for the same sample mounting (so the same $y(z)$-relation), the change in piezo voltage required to change $R_{T}$ from $1 \mathrm{G} \Omega$ to $100 \mathrm{k} \Omega$ could occasionally be up to $50 \%$ larger than usual for that specific mounting.

Qualitatively, the experimental results seem to support the theoretical work of Laloyaux et al. [13]. However, the actual shapes of the electrodes were not known in these experiments.

For many tunneling experiments, and certainly for experiments which intend to study effects of the

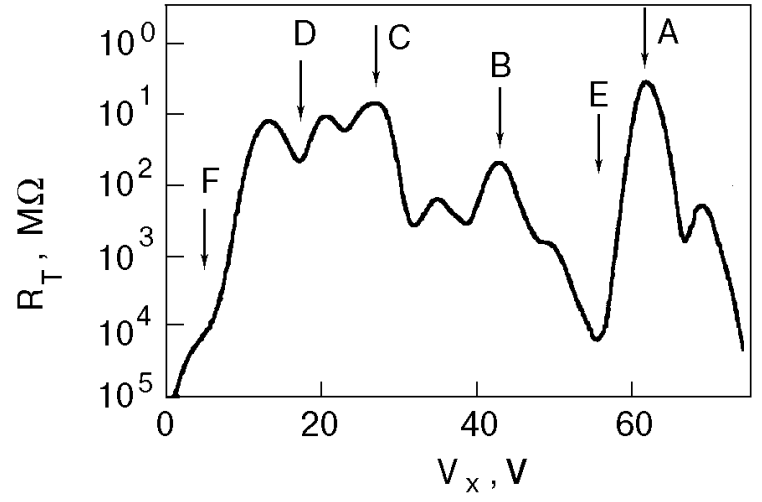

( lateral displacement )

Fig. 5. Logarithmic plot of the tunnel resistance of a Pt MCB junction as a function of the shear-piezo voltage $(T=4.2 \mathrm{~K}$, $V_{\text {bias }}=50 \mathrm{mV}$ ). The displacement is estimated to be $0.5-1.0 \AA / \mathrm{V}$. Assuming one of the electrodes to be atomically sharp, the curve can be interpreted as a line profile of the other electrode, demonstrating that the surface is rather rough. The arrows indicate different types of selected locations at which $\log \left(R_{T}(z)\right)$ were recorded (see text).

electrode geometry, it is useful to have at least some knowledge of the electrode surface topography. We therefore also performed experiments where small pieces $(5 \times 3 \times 1 \mathrm{~mm})$ of piezo material were placed underneath the two anchoring points. Using one piece that changes its thickness as a voltage is applied, and one piece that gives a horizontal displacement of its surfaces with respect to each other (shear piezo), it was possible to move the electrodes with respect to each other laterally. The variation of the tunnel current can then be measured as the electrodes are moved around. The obtained lateral displacement at $4.2 \mathrm{~K}$ is estimated to be about $0.6 \AA$ 部.

The breaking process is likely to result in at least one electrode being sharp, and using this electrode as a tip, a three dimentional scan of the other (more blunt) electrode can be obtained. A typical example is given in Fig. 4 (see also Ref. 15). It show features that can be interpreted as atomically resolved images and demonstrates that the surface of the ( $\mathrm{Au}-)$ electrodes is rather rough, which can be expected for electrodes created in a breaking process.

The shear-piezo option was used to record $\log \left(R_{T}(z)\right)$ curves for several different Pt samples at selected sites such as the ones indicated in Fig. 5. Assuming that a shear-piezo scan like Fig. 5 represents a profile of the «blunt» electrode, while the other electrode acts as a sharp tip, the $\log \left(R_{T}(z)\right)$ curves recorded at the selected sites are expected to correspond to the theoretical situations [13] of a tip opposing a boss (Fig. 5, locations $A$ and $B$ ), a 


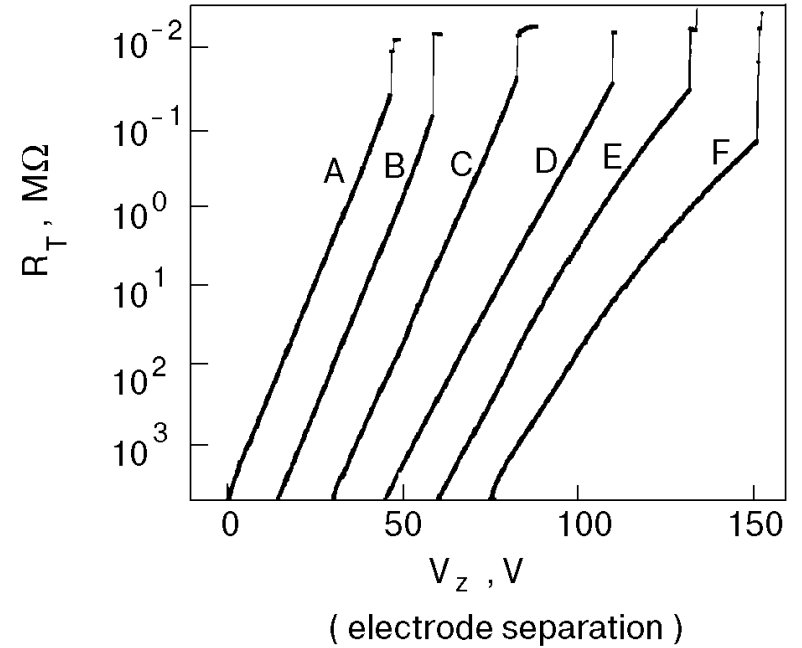

Fig. 6. Logarithmic plot of the tunnel resistance of Pt MCB junctions as a function of $z$-piezo voltage $\left(T=4.2 \mathrm{~K}, V_{\text {bias }}=\right.$ $=5 \mathrm{mV})$. Curves $A-F$ are examples of the $\log \left(R_{T}(z)\right)$ behavior that was mostly observed for different samples at selected locations comparable to the locations $A-F$ indicated in Fig. 5 .

$\operatorname{dip}(E)$, or a rather flat part of the surface ( $C$ and $D)$. Figure 6 shows six $\log \left(R_{T}(z)\right)$ curves representing the behavior that was generally observed when these curves were recorded at selected locations that are similar to the sites $A-F$ indicated in Fig. 5. Bended $\log \left(R_{T}(z)\right)$ curves were mostly only found for locations like $E$ and $F$ in Fig. 5, the bending being stronger for $F$, where the «tip» is approaching a very steep part of the «sample», and several tip atoms may contribute to the electron tunneling process. At locations like $A, B, C$, and $D$, mainly straight $\log \left(R_{T}(z)\right)$ curves were obtained, the curves at $D$-type locations often being less steep than for $A-C$. Still, bended curves were sometimes observed for locations $B, C$ and $D$, but for very sharp locations like $A$, the $\log \left(R_{T}(z)\right)$ curves were almost always straight lines. These experimental results do not correspond to the ones that were expected from the work of Laloyaux et al. [13]. The experiment was repeated using $\mathrm{Al}$ and $\mathrm{Pt}-\mathrm{Ir} \mathrm{MCB}$ junctions. In general, the same discrepancy between theoretical predictions and experimental results was found. Since the experimental situation is far more complicated than the theoretical one, where planar surfaces with cylindrically symmetric protrusions and indentations of a certain shape were considered, the model geometries may be too simple to describe adequately the tunneling between the rather rough electrode surfaces of an MCB junction. Also, the information on the surface topography that we have is limited to only a single line; what seems to be a flat part in our line scan, might turn out to be not flat at all if the perpendicular direction would be scanned also. Additionally, one should bear in mind that the line scan of Fig. 5 cannot be an exact image of the surface topography, because the slope of the curves differs for different locations, and because the $\log \left(R_{T}(z)\right)$ curves are sometimes bended. Nevertheless, since it is not likely that the shape and slope of the $\log \left(R_{T}(z)\right)$ curve changes abruptly somewhere along the scanned line, and because these changes are much smaller than the ones displayed in Fig. 5, the recorded line scan will still provide a reasonable image of the surface topography in one direction. The measurements at selected locations therefore show that at locations with different surface topographies, different $\log \left(R_{T}(z)\right)$ relations may be found.

We also studied the jump to contact at locations like $A-F$ of Fig. 5. The behavior of one-atom contacts has been studied extensively by Krans et al. [12]. In experiments where the contact size of MCB junctions, was slowly decreased, they found that $\mathrm{Al}$ and $\mathrm{Cu}$ tend to jump to tunneling from a conductance value close to $2 e^{2} / h$, the conductance quantum for a single conductance channel. Pt junctions, however, were found to jump mostly from a 1.4-1.8 times larger value, indicating that more than one conductance channel was present shortly before the jump. This was explained in terms of $s$ and $d$-like wave functions, constituting separate conductance channels for Pt.

The line scan was used to do a site-selected investigation of the jump to contact for Pt MCB junctions. At locations comparable to $E$ and $F$ in Fig. 5, jumps to what seemed to be multi-atom contacts usually occurred. At locations like $B$ and $C$, there were mostly immediate jumps to a conductance value of 1.5-2.0 conductance quanta (so comparable to the results of Ref. 12), while at $D$, there were jumps to a much broader range of resistance values, corresponding to 1.5 or more conductance quanta. The most remarkable result was found at very sharp locations like $A$ in Fig. 5. At such locations, a jump to a resistance value close to $h / 2 e^{2}$ (so only one conductance channel present), quickly followed by a jump to about half the resistance value (two conductance channels), was observed for about $60 \%$ of all cases. An example of this type of behavior is the jump to contact of curve $A$ in Fig. 6. These results demonstrate that the specific electrode geometry also influences the way in which the first conductance channels are formed when the electrodes are slowly brought into contact. 


\section{Conclusion}

In the above it has been shown experimentally that the tunnel resistance as a function of the electrode spacing can deviate strongly from the expected exponential behavior over the whole experimental range of more than four orders of magnitude of the tunnel resistance. From comparison with previous theoretical studies can be concluded that these deviations are not likely to be due to interactions between the electrodes, but can be attributed to effects of the tip-sample geometry on the dependence of the tunnel current on the distance between the two electrodes. Qualitatively, the results correspond to the calculations of Laloyaux et al.; however, tunneling at selected locations using a line profile of one of the electrodes indicates that the rough surfaces of an MCB junction cannot be completely described by the rather straightforward theoretical model.

Part of this work was supported by the Stichting voor Fundamenteel Onderzoek der Materie (FOM) which is financially supported by the Nederlandse Organisatie voor Weten-schappelijk Onderzoek (NWO). We thank J. G. H. Hermsen, J. W. Gerritsen, and E. J. G. Boon for technical and computational support. O. I. S. wishes to acknowledge the NWO for a visitor's grant.
1. L. L. Soethout, H. van Kempen, and G. F. A. van de Walle, Adv. Electron. Electron Phys. 79, 155 (1990).

2. L. E. C. van de Leemput and H. van Kempen, Rep. Prog. Phys. 55, 1165 (1992).

3. Scanning Tunneling Microscopy I, Springer Series in Surface Sciences 20, H.-J. Güntherodt and R. Wiesendanger (eds.), Springer Verlag, Berlin (1992).

4. Scanning Tunneling Microscopy II, Springer Series in Surface Sciences 28, R. Wiesendanger and H.-J. Güntherodt (eds.), Springer Verlag, Berlin (1992).

5. Scanning Tunneling Microscopy III, Springer Series in Surface Sciences 29, R. Wiesendanger and H.-J. Güntherodt (eds.), Springer Verlag, Berlin (1993).

6. J. G. Simmons, J. Appl. Phys. 34, 1793 (1963).

7. J. K. Gimzewski and R. Möller, Phys. Rev. B36, 1284 (1987); J. K. Gimzewski, R. Möller, D. W. Pohl, and R. R. Schlittler, Surf. Science 189/190, 15 (1987).

8. N. D. Lang, Phys. Rev. B36, 8173 (1987).

9. J. Ferrer, A. Martín-Rodero, and F. Flores, Phys. Rev. B38, 10113 (1988).

10. U. Dürig, O. Züger, and D. W. Pohl, Phys. Rev. Lett. 65 349 (1990).

11. S. Ciraci and E. Tekman, Phys. Rev. B40, 11969 (1989).

12. J. M. Krans, C. J. Muller, I. K. Yanson, Th. C. M. Govaert, R. Hesper, and J. M. van Ruitenbeek, Phys. Rev. B48, 14721 (1993).

13. Th. Laloyaux, I. Derycke, J.-P. Vigneron, Ph. Lambin, and A. A. Lucas, Phys. Rev. B47, 7508 (1993).

14. S. Ciraci, E. Tekman, A. Baratoff, and I. P. Batra, Phys. Rev. B46, 10411 (1992).

15. J. Voets, R. J. P. Keijsers, O. I. Shklyarevskii, and H. van Kempen, Phys. Rev. B53, 1072 (1996)

16. C. J. Muller, J. M. van Ruitenbeek, and L. J. de Jongh, Physica C 191, 485 (1992).

17. W. C. Young, Roark's Formulas for Stress and Strain McGraw-Hill, New York (1989), ch. 7. 